\title{
Balance Between Allylic C-H Activation and Cyclopropanation in the Reactions of Donor/Acceptor-Substituted Rhodium Carbenoids with Trans Alkenes
}

\author{
Huw M. L. Davies, * Michael G. Coleman and Dominic L. Ventura \\ Department of Chemistry, University at Buffalo, The State University of New York, \\ Buffalo, New York 14260-3000.
}

Supporting Information 
General Methods: ${ }^{1} \mathrm{H}$ NMR spectra were run at either 300,400 , or $500 \mathrm{MHz}$, and ${ }^{13} \mathrm{C}$ NMR at either 75 or $125 \mathrm{MHz}$ in $\mathrm{CDCl}_{3}$ unless otherwise noted. Mass spectral determinations were carried out with Magneticsector from Thermofinnigan MAT95XL, positive electrospray ionization with capillary temperature $250{ }^{\circ} \mathrm{C}$ and spray voltage 2.7 $\mathrm{kV}$. Melting points were uncorrected. IR spectra were obtained using a Nicolet Impact series 420 IR. Optical rotations were measured using a Jaso DIP-370 digital polarimeter. Glassware was flame dried prior to use. 2,2-Dimethylbutane (DMB) was distilled from calcium hydride. Solvents were dried using a solvent purifier. Analytical thin layer chromatography (TLC) was performed on Whatman Reagent $250 \mu$ m layer silica gel aluminum backed plates with visualization by ultraviolet (UV) irradiation at $254 \mathrm{nM}$ and/or staining with potassium permanganate, phosphomolybdic acid, or ninhydrin. The eluting system for each purification was determined by TLC analysis. All the organic solvents were removed under reduced pressure using a rotary evaporator. Chromatography solvents were used without distillation. Column chromatography was carried out on Merck silica gel $60(230$ - 400) mesh. Reactions were carried out under an atmosphere of argon. Elemental analysis was performed by Atlantic Microlab, Inc.; Norcoss, Georgia. Column chromatography was carried out on ICN SiliTech 32-63D 60 $\AA$. Enantiomeric excess was determined by HPLC using Chiracel OD-H, $(R, R)$-Whelk-O 1 or $(S, S)$-Whelk-O 1 chiral analytical columns (UV detection at $254 \mathrm{~nm}$ ). Commercially available reagents were used without additional purification unless noted. The known compounds $\quad \mathrm{Rh}_{2}(\mathrm{~S} \text {-DOSP) })_{4},{ }^{1} \quad$ (E)-1-(but-1-enyl)-4-methoxybenzene $\quad \mathbf{1}^{2}{ }^{2}$ methyl phenyldiazoacetates $\mathbf{2 a - f}$, $^{3-6}$ phenylvinyl diazoacetate $7,{ }^{7}$ and alkenes $\mathbf{9 b - \mathbf { d } ^ { 8 , 9 }}$ were all 
prepared by literature procedures and the spectral data showed good agreement with the literature data.

\section{General procedure for Rhodium(II)-Catalyzed Decompositions of Methyl} Phenyldiazoacetates in the Presence of trans-Alkenes: In a dry $25 \mathrm{~mL}$ round bottom flask the trans-alkene (1.0 eq.) and Rh(II) catalyst (0.02 eq.) were dissolved in 2,2dimethylbutane (DMB) $(10 \mathrm{~mL})$. The diazo (2.0 eq.) was stirred in distilled DMB (20 $\mathrm{mL}$ ) under Ar for $5 \mathrm{~min}$. It was then added via syringe pump for a $3 \mathrm{~h}$ period. The solution was then allowed to stir for an additional $2 \mathrm{~h}$. The solution was concentrated, dry-mounted on silica, and subjected to flash chromatography using pentane/diethyl ether as the eluent in the percentage specified in parenthesis.

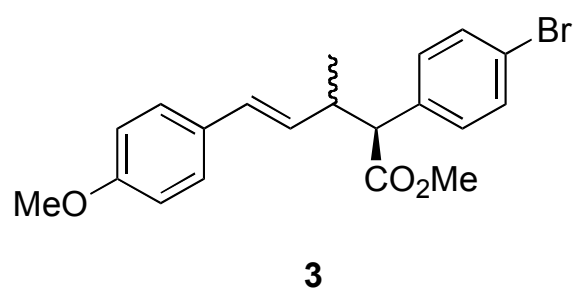

\section{(S,E)-Methyl 2-(4-bromophenyl)-5-(4-methoxyphenyl)-3-methylpent-4-enoate (3)}

In a $25 \mathrm{~mL}$ round bottom flask (E)-1-(but-1-enyl)-4-methoxybenzene 1 (162 mg, 1.00 mmol, 1.00 eq.) and $\mathrm{Rh}_{2}(S \text {-DOSP })_{4}(38 \mathrm{mg}, 0.02 \mathrm{mmol}, 0.02$ eq. $)$ were dissolved in $\mathrm{DMB}$ (6 mL). Diazo 2a (508 mg, $2.00 \mathrm{mmol}, 2.00$ eq.) was stirred in distilled DMB (12 mL) under Ar for $5 \mathrm{~min}$. It was then added via syringe pump for a $3 \mathrm{~h}$ period. The solution was then allowed to stir for an additional $2 \mathrm{~h}$. The solution was concentrated and it was determined by crude ${ }^{1} \mathrm{H}$ NMR that a $1: 1.37$ diastereomeric mixture resulted. The residue was purified by flash chromatography $\left(\mathrm{SiO}_{2}, 20 \% \mathrm{Et}_{2} \mathrm{O} /\right.$ pentane $)$ to give clear oil 3 as an inseparable mixture of diastereomers $(349 \mathrm{mg}, 0.897 \mathrm{mmol}, 90 \%$ yield $) . \mathrm{R}_{\mathrm{f}}=0.21$ 
$\left(20 \% \mathrm{Et}_{2} \mathrm{O} /\right.$ pentane). ${ }^{1} \mathrm{H}$ NMR $\left(400 \mathrm{MHz}, \mathrm{CDCl}_{3}\right) \delta 7.47(\mathrm{~d}, J=8 \mathrm{~Hz}, 2 \mathrm{H}), 7.40(\mathrm{~d}, J=8$ Hz, 2H), 7.27 (m, H), 7.19 (d, $J=8 \mathrm{~Hz}, 2 \mathrm{H}), 7.09$ (d, $J=8 \mathrm{~Hz}, 2 \mathrm{H}), 6.84$ (d, $J=8 \mathrm{~Hz}$, 2H) $6.76(\mathrm{~d}, J=8 \mathrm{~Hz}, 2 \mathrm{H}), 6.42(\mathrm{~d}, J=16 \mathrm{~Hz}, 1 \mathrm{H}), 6.14(\mathrm{~d}, J=16 \mathrm{~Hz}, 1 \mathrm{H}), 5.96(\mathrm{~m}$, 1H), $5.68(\mathrm{~m}, 1 \mathrm{H}), 3.80(\mathrm{~s}, 3 \mathrm{H}), 3.77(\mathrm{~s}, 3 \mathrm{H}), 3.68(\mathrm{~s}, 3 \mathrm{H}), 3.57(\mathrm{~s}, 3 \mathrm{H}), 3.39(\mathrm{~m}, 2 \mathrm{H})$, $2.99(\mathrm{~m}, 2 \mathrm{H}), 1.19(\mathrm{~d}, J=7 \mathrm{~Hz}, 3 \mathrm{H}), 0.88(\mathrm{~d}, J=7 \mathrm{~Hz}, 3 \mathrm{H}) .{ }^{13} \mathrm{C} \mathrm{NMR}\left(75 \mathrm{MHz}, \mathrm{CDCl}_{3}\right)$ $\delta 173.4,173.2,159.0,158.9,136.5,136.4,131.6,131.4,130.4,130.3,130.1,130.0$, $129.9,129.9,129.7,129.6,127.3,127.1,121.4,121.3,113.9,113.8,57.9,57.8,55.2$, 55.2, 51.9, 51.8, 41.0, 40.3, 19.1, 18.2. IR (neat): 1734, 1608, 1511, 1488, 1247, 1175, $1159,1073,1034,1101,817 \mathrm{~cm}^{-1}$. HPLC analysis: $81 \%$ ee minor diastereomer and $94 \%$ ee major diastereomer (OD-H, $0.5 \% i-\mathrm{PrOH}$ in hexane, $0.5 \mathrm{~mL} / \mathrm{min}, \lambda=254 \mathrm{nM}$, minor diastereomer: $t_{R}=25.2$ min, minor, $t_{R}=26.7$ min, major; major diastereomer: $t_{R}=28.5$ min, minor, $t_{R}=30.1$ min major). HRMS (ESI) Calcd for $\left[\mathrm{C}_{20} \mathrm{H}_{21} \mathrm{BrO}_{3}\right]^{+} \mathrm{Na}\left(\mathrm{M}^{+} \mathrm{Na}\right)$ : 411.0566. Found: 411.0572.

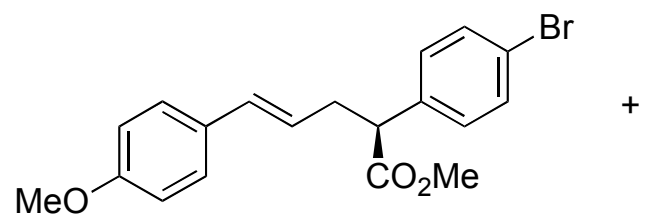

$5 a$

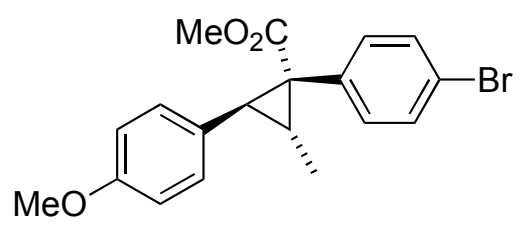

$6 a$

$(S, E)-M e t h y l ~ 2-(4-b r o m o p h e n y l)-5-(4-m e t h o x y p h e n y l) p e n t-4-e n o a t e ~(5 a)$ and (1R,2S,3R)-Methyl 1-(4-bromophenyl)-2-(4-methoxyphenyl)-3methylcyclopropanecarboxylate (6a):

In a $25 \mathrm{~mL}$ round bottom flask trans-anethole, $4(534 \mathrm{mg}, 3.60 \mathrm{mmol}, 1.00$ eq.) and $\mathrm{Rh}_{2}(S \text {-DOSP })_{4}(13 \mathrm{mg}, 0.07 \mathrm{mmol}, 0.02$ eq. $)$ were dissolved in DMB (18 mL). Diazo 2a (1.87 g, $7.37 \mathrm{mmol}, 2.05$ eq.) was stirred in distilled DMB (36 mL) under Ar for 5 min. 
The solution of 2a was then added via syringe pump over a $3 \mathrm{~h}$ period and the mixture was stirred for an additional $2 \mathrm{~h}$. The solution was concentrated and analysis of the crude ${ }^{1} \mathrm{H}$ NMR spectrum indicated a product ratio of $(3: 1 ; \mathbf{5 a}: \mathbf{6 a})$. The residue was purified by flash chromatography $\left(\mathrm{SiO}_{2}, 20 \% \mathrm{Et}_{2} \mathrm{O} /\right.$ pentane) to give $\mathbf{5 a}(589 \mathrm{mg}, 1.57 \mathrm{mmol}, 44 \%$ yield) as a white solid and $\mathbf{6 a}(291 \mathrm{mg}, 0.78 \mathrm{mmol}, 22 \%$ yield) as a clear oil.

Compound 5a: White solid, mp $81{ }^{\circ} \mathrm{C} . \quad \mathrm{R}_{\mathrm{f}}=0.29\left(20 \% \mathrm{Et}_{2} \mathrm{O} /\right.$ pentane $) . \quad[\alpha]_{\mathrm{D}}^{23}=-109.4$ $\left(\mathrm{c}=1.0, \mathrm{CHCl}_{3}\right) .{ }^{1} \mathrm{H} \mathrm{NMR}\left(500 \mathrm{MHz}, \mathrm{CDCl}_{3}\right) \delta 7.44(\mathrm{~d}, J=8 \mathrm{~Hz}, 2 \mathrm{H}), 7.21(\mathrm{~m}, 4 \mathrm{H})$, $6.82(\mathrm{~d}, J=8 \mathrm{~Hz}, 2 \mathrm{H}), 6.35(\mathrm{~d}, J=16 \mathrm{~Hz}, 2 \mathrm{H}), 5.91(\mathrm{dt}, J=8 \mathrm{~Hz}, 16 \mathrm{~Hz}, 1 \mathrm{H}), 3.77$ (s, 3H), $3.66(\mathrm{~m}, 4 \mathrm{H}), 2.89(\mathrm{~m}, 1 \mathrm{H}), 2.63(\mathrm{~m}, 1 \mathrm{H}) .{ }^{13} \mathrm{C} \mathrm{NMR}\left(75 \mathrm{MHz}, \mathrm{CDCl}_{3}\right) \delta$ 173.4, $158.9,137.5,131.9,131.7,129.9,129.7,127.2,124.0,121.3,113.9,55.2,52.0,51.3$, 36.8. IR (neat): $1733,1511,1247,1174,1161,907,729 \mathrm{~cm}^{-1}$. HPLC analysis: $93 \%$ ee (OD-H, $0.5 i$-PrOH in hexane, $1.0 \mathrm{~mL} / \mathrm{min}, \lambda=254 \mathrm{nM}, \mathrm{t}_{\mathrm{R}}=22.6$ min, major, $\mathrm{t}_{\mathrm{R}}=25.6$ min, minor). Anal. Calcd for $\mathrm{C}_{19} \mathrm{H}_{19} \mathrm{BrO}_{3}: \mathrm{C}, 60.81 ; \mathrm{H}$, 5.10. Found: $\mathrm{C}, 60.97 ; \mathrm{H}, 5.20$. HRMS (EI) Calcd for $\mathrm{C}_{19} \mathrm{H}_{19} \mathrm{BrO}_{3}$ (M): 374.0512. Found: 374.0518 .

Compound 6a: Clear oil. $\mathrm{R}_{\mathrm{f}}=0.33\left(20 \% \mathrm{Et}_{2} \mathrm{O} /\right.$ pentane $) . \quad[\alpha]_{\mathrm{D}}{ }^{23}=+85.8(\mathrm{c}=1.0$, $\left.\mathrm{CHCl}_{3}\right) ;{ }^{1} \mathrm{H}$ NMR $\left(500 \mathrm{MHz}, \mathrm{CDCl}_{3}\right) 7.24(\mathrm{~m}, 2 \mathrm{H}), 6.86(\mathrm{~d}, J=8 \mathrm{~Hz}, 2 \mathrm{H}), 6.63(\mathrm{~m}, 4 \mathrm{H})$, $3.71(\mathrm{~s}, 3 \mathrm{H}), 3.63(\mathrm{~s}, 3 \mathrm{H}), 3.03(\mathrm{~d}, J=8 \mathrm{~Hz}, 1 \mathrm{H}), 2.13(\mathrm{~m}, 1 \mathrm{H}), 1.45(\mathrm{~d}, J=7 \mathrm{~Hz}, 3 \mathrm{H})$. ${ }^{13} \mathrm{C}$ NMR $\left(75 \mathrm{MHz}, \mathrm{CDCl}_{3}\right) \delta 171.9,158.1,135.8,133.1,130.9,128.9,128.5,120.9$, 113.4, 55.1, 52.3, 42.3, 37.4, 27.5, 12.7. IR (neat): 1716, 1516, 1249, 1169, 1101, 907, $728 \mathrm{~cm}^{-1}$. HPLC analysis: $85 \%$ ee $((R, R)$-Whelk, $0.5 i$-PrOH in hexane, $1.0 \mathrm{~mL} / \mathrm{min}, \lambda=$ $254 \mathrm{nM}, \mathrm{t}_{\mathrm{R}}=6.6 \mathrm{~min}$, minor, $\mathrm{t}_{\mathrm{R}}=8.8$ min, major). Anal. Calcd for $\mathrm{C}_{19} \mathrm{H}_{19} \mathrm{BrO}_{3}: \mathrm{C}$, 
60.81; H, 5.10. Found: $\mathrm{C}, 61.05 ; \mathrm{H}, 5.18$. HRMS (EI) Calcd for $\mathrm{C}_{19} \mathrm{H}_{19} \mathrm{BrO}_{3}$ (M): 374.0522. Found: 374.0525.

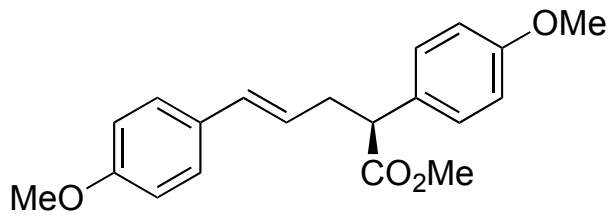

$5 b$

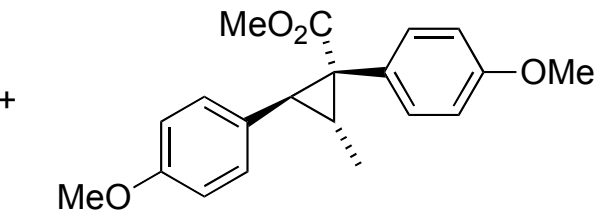

6b

$(S, E)$-Methyl 2,5-bis(4-methoxyphenyl)pent-4-enoate (5b) and (1R,2S,3R)-Methyl 1,2-bis(4-methoxyphenyl)-3-methylcyclopropanecarboxylate (6b)

In a $25 \mathrm{~mL}$ round bottom flask trans-anethole $4(150 \mathrm{mg}, 1.00 \mathrm{mmol}, 1.00$ eq.) and $\mathrm{Rh}_{2}(S \text {-DOSP) })_{4}(38 \mathrm{mg}, 0.02 \mathrm{mmol}, 0.02$ eq.) were dissolved in DMB (6 mL). Diazo $2 \mathbf{b}$ (417 mg, $2.00 \mathrm{mmol}, 2.00$ eq.) was stirred in distilled DMB (12 mL) under Ar for $5 \mathrm{~min}$. It was then added via syringe pump for a $3 \mathrm{~h}$ period. The solution was then allowed to stir for an additional $2 \mathrm{~h}$. The solution was concentrated and crude ${ }^{1} \mathrm{H}$ NMR spectra indicated a product ratio of $(8: 1 ; \mathbf{5 b}: \mathbf{6 b})$. The residue was then purified by flash chromatography $\left(\mathrm{SiO}_{2}, 20 \% \mathrm{Et}_{2} \mathrm{O} /\right.$ pentane) to give $\mathbf{5 b}$ ( $221 \mathrm{mg}, 0.68 \mathrm{mmol}, 67 \%$ yield) as a white solid and $\mathbf{6 b}(17 \mathrm{mg}, 0.052 \mathrm{mmol}, 5 \%$ yield) as a white solid.

Compound 5b: White solid, $\mathrm{mp} 83^{\circ} \mathrm{C} . \quad \mathrm{R}_{\mathrm{f}}=0.20\left(20 \% \mathrm{Et}_{2} \mathrm{O} /\right.$ pentane $) .[\alpha]_{\mathrm{D}}{ }^{23}=-82.4(\mathrm{c}$ $\left.=1.0, \mathrm{CHCl}_{3}\right) .{ }^{1} \mathrm{H} \operatorname{NMR}\left(500 \mathrm{MHz}, \mathrm{CDCl}_{3}\right) \delta 7.24(\mathrm{~m}, 4 \mathrm{H}), 6.86(\mathrm{~d}, J=9 \mathrm{~Hz}, 2 \mathrm{H}) 6.81$ (d, $J=9 \mathrm{~Hz}, 2 \mathrm{H}), 6.35(\mathrm{~d}, J=16 \mathrm{~Hz}, 1 \mathrm{H}), 5.94(\mathrm{dt}, J=7 \mathrm{~Hz}, 16 \mathrm{~Hz}, 1 \mathrm{H}), 3.78(\mathrm{~d}, 6 \mathrm{H})$, $3.63(\mathrm{~m}, 4 \mathrm{H}), 2.91(\mathrm{~m}, 1 \mathrm{H}), 2.60(\mathrm{~m}, 1 \mathrm{H}) .{ }^{13} \mathrm{C} \mathrm{NMR}\left(75 \mathrm{MHz}, \mathrm{CDCl}_{3}\right) \delta$ 174.2, 158.9, $158.8,131.5,130.7,130.2,128.9,127.2,124.8,114.1,113.9,55.2,51.9,51.1,37.1$. IR (neat): 1733, 1608, 1583, 1517, 1463, 1439, 1302, 1249, 1176, 1158, 1109, 1034, 968, 
832, $800 \mathrm{~cm}^{-1}$. HPLC analysis: 79\% ee (OD-H, $2.0 i$-PrOH in hexane, $0.8 \mathrm{~mL} / \mathrm{min}, \lambda=$ $254 \mathrm{nM}, \mathrm{t}_{\mathrm{R}}=13.7$ min, minor, $\mathrm{t}_{\mathrm{R}}=15.9$ min, major). Anal. Calcd for $\mathrm{C}_{20} \mathrm{H}_{20} \mathrm{O}_{3}: \mathrm{C}$, 73.60; H, 6.79. Found: C, 73.54; H, 6.85. HRMS (ESI) Calcd for $\left[\mathrm{C}_{20} \mathrm{H}_{22} \mathrm{O}_{4}\right]^{+} \mathrm{Na}\left(\mathrm{M}^{+} \mathrm{Na}\right)$ : 349.1410. Found: 349.1404 .

Compound 6b: White solid, $\mathrm{mp} 84{ }^{\circ} \mathrm{C} . \quad \mathrm{R}_{\mathrm{f}}=0.17\left(20 \% \mathrm{Et}_{2} \mathrm{O} /\right.$ pentane $) .[\alpha]_{\mathrm{D}}{ }^{23}=-219.2$ $\left(\mathrm{c}=2.1, \mathrm{CHCl}_{3}\right) .{ }^{1} \mathrm{H} \mathrm{NMR}\left(500 \mathrm{MHz}, \mathrm{CDCl}_{3}\right) 6.90(\mathrm{~d}, J=8 \mathrm{~Hz}, 2 \mathrm{H}), 6.67(\mathrm{~d}, J=8 \mathrm{~Hz}$, 4H), $6.61(\mathrm{~d}, J=9 \mathrm{~Hz}, 2 \mathrm{H}), 3.73(\mathrm{~s}, 3 \mathrm{H}), 3.70(\mathrm{~s}, 3 \mathrm{H}), 3.64(\mathrm{~s}, 3 \mathrm{H}), 2.98(\mathrm{~d}, J=8 \mathrm{~Hz}$, 1H), $2.11(\mathrm{~m}, 1 \mathrm{H}), 1.44(\mathrm{~d}, J=6 \mathrm{~Hz}, 3 \mathrm{H}) .{ }^{13} \mathrm{C} \mathrm{NMR}\left(75 \mathrm{MHz}, \mathrm{CDCl}_{3}\right) \delta 172.7,158.3$, $157.9,132.4,129.2,128.9,128.6,113.2,113.1,55.1,55.0,52.3,42.2,37.3,27.8,12.6$. IR (neat): 1732, 1608, 1509, 1245, 1175, 1157, 1032, 967, 831, $800 \mathrm{~cm}^{-1}$. HPLC analysis: $82 \%$ ee $\left(\mathrm{OD}-\mathrm{H}, 0.5 i-\mathrm{PrOH}\right.$ in hexane, $1.0 \mathrm{~mL} / \mathrm{min}, \lambda=254 \mathrm{nM}, \mathrm{t}_{\mathrm{R}}=14.0 \mathrm{~min}$, major, $\mathrm{t}_{\mathrm{R}}=22.3$ min, minor). Anal. Calcd for $\mathrm{C}_{20} \mathrm{H}_{20} \mathrm{O}_{3}: \mathrm{C}, 73.60 ; \mathrm{H}, 6.79$. Found: $\mathrm{C}$, 73.41; $\mathrm{H}, 6.94$. HRMS (EI) Calcd for $\mathrm{C}_{20} \mathrm{H}_{22} \mathrm{O}_{4}$ (M): 326.1513. Found: 326.1516 .

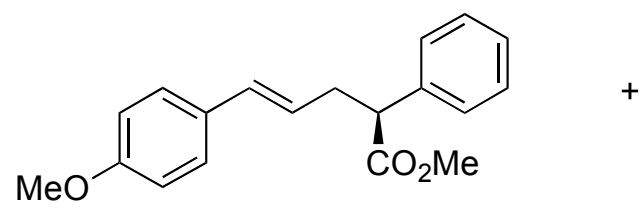

$5 c$

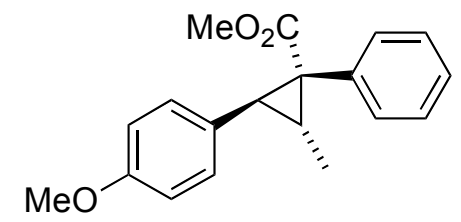

6c

(S,E)-Methyl 5-(4-methoxyphenyl)-2-phenylpent-4-enoate (5c) and $(1 R, 2 S, 3 R)$-Methyl 2-(4-methoxyphenyl)-3-methyl-1-phenylcyclopropanecarboxylate (6c)

In a dry $25 \mathrm{~mL}$ round bottom flask trans-anethole $4(150 \mathrm{mg}, 1.00 \mathrm{mmol}, 1.00 \mathrm{eq}$.$) and$ $\mathrm{Rh}_{2}(S \text {-DOSP })_{4}(38 \mathrm{mg}, 0.02 \mathrm{mmol}, 0.02$ eq.) were dissolved in DMB $(6 \mathrm{~mL})$. Diazo 2c ( $356 \mathrm{mg}, 2.00 \mathrm{mmol}, 2.00$ eq.) was stirred in distilled DMB (12 mL) under Ar for $5 \mathrm{~min}$ 
and then added to the reaction via syringe pump over a $3 \mathrm{~h}$ period. The solution was then allowed to stir for an additional $2 \mathrm{~h}$, concentrated, and crude ${ }^{1} \mathrm{H}$ NMR spectrum indicated a product ratio of $(8: 1 ; \mathbf{5 c}: \mathbf{6 c})$. The residue was purified by flash chromatography $\left(\mathrm{SiO}_{2}, 20 \% \mathrm{Et}_{2} \mathrm{O} /\right.$ pentane) to give $\mathbf{5 c}(158 \mathrm{mg}, 0.53 \mathrm{mmol}, 53 \%$ yield $)$ as a white solid and $6 \mathbf{c}$ (24 mg, $0.08 \mathrm{mmol}, 8 \%$ yield) as a white solid.

Compound 5c: White solid, mp $72{ }^{\circ} \mathrm{C} . \quad \mathrm{R}_{\mathrm{f}}=0.29\left(20 \% \mathrm{Et}_{2} \mathrm{O} /\right.$ pentane $) . \quad[\alpha]_{\mathrm{D}}^{23}=-87.4(\mathrm{c}$ $\left.=1.0, \mathrm{CHCl}_{3}\right) .{ }^{1} \mathrm{H} \mathrm{NMR}\left(500 \mathrm{MHz}, \mathrm{CDCl}_{3}\right) \delta 7.26(\mathrm{~m}, 7 \mathrm{H}), 6.80(\mathrm{~d}, J=9 \mathrm{~Hz}, 2 \mathrm{H}), 6.36$ (d, $J=16 \mathrm{~Hz}, 1 \mathrm{H}), 5.95$ (dt, $J=7 \mathrm{~Hz}, 16 \mathrm{~Hz}, 1 \mathrm{H}), 3.78$ (s, 3H), 3.69 (dd, $J=7 \mathrm{~Hz}, 9 \mathrm{~Hz}$, 1H) $3.65(\mathrm{~s}, 3 \mathrm{H}), 2.95(\mathrm{~m}, 1 \mathrm{H}), 2.63(\mathrm{~m}, 1 \mathrm{H}) .{ }^{13} \mathrm{C} \mathrm{NMR}\left(125 \mathrm{MHz}, \mathrm{CDCl}_{3}\right) \delta$ 173.9, $158.9,138.6,131.6,130.1,128.7,127.9,127.3,127.2,124.6,113.8,55.2,51.9,51.9$, 37.0. IR (neat): 1737, 1609, 1513, 1438, 1252, 1162, 1023, 964, 847, 799, 736, $704 \mathrm{~cm}^{-1}$. HPLC analysis: $91 \%$ ee $((R, R)$-Whelk, $5 \% i$-PrOH in hexane, $1.0 \mathrm{~mL} / \mathrm{min}, \lambda=254 \mathrm{nM}$, $t_{R}=10.1$ min, minor, $t_{R}=11.1$ min, major). Anal. Calcd for $\mathrm{C}_{19} \mathrm{H}_{20} \mathrm{O}_{3}: \mathrm{C}, 77.00 ; \mathrm{H}, 6.80$. Found: C, 76.77; H, 6.76. HRMS (EI) Calcd for $\mathrm{C}_{19} \mathrm{H}_{20} \mathrm{O}_{3}$ (M): 296.1407. Found: 296.1409.

Compound 6c: White solid, mp $83{ }^{\circ} \mathrm{C} . \mathrm{R}_{\mathrm{f}}=0.31\left(20 \% \mathrm{Et}_{2} \mathrm{O} /\right.$ pentane $) .[\alpha]_{\mathrm{D}}^{23}=-125.8(\mathrm{c}$ $\left.=1.0, \mathrm{CHCl}_{3}\right) .{ }^{1} \mathrm{H} \mathrm{NMR}\left(500 \mathrm{MHz}, \mathrm{CDCl}_{3}\right) \delta 7.13(\mathrm{~m}, 3 \mathrm{H}), 7.00(\mathrm{~m}, 2 \mathrm{H}), 6.66(\mathrm{~d}, J=8$ Hz, 2H), 6.59 (d, $J=8 \mathrm{~Hz}, 2 \mathrm{H}), 3.69$ (s, 3H), 3.64 (s, 3H), 3.02 (d, $J=8 \mathrm{~Hz}, 1 \mathrm{H}), 2.17$ $(\mathrm{m}, 1 \mathrm{H}), 1.45(\mathrm{~d}, J=6 \mathrm{~Hz}, 3 \mathrm{H}) .{ }^{13} \mathrm{C} \mathrm{NMR}\left(75 \mathrm{MHz}, \mathrm{CDCl}_{3}\right) \delta 172.5,157.9,136.5$, $131.4,129.0,128.9,127.8,126.8,113.2,55.1,52.3,43.0,30.9,27.4,12.7$. IR (neat): 1733, 1511, 1246, 1215, 1167, 1033, 967, 838, 733, $699 \mathrm{~cm}^{-1}$. HPLC analysis: $80 \%$ ee (OD-H, $0.5 \% i$-PrOH in hexane, $1.0 \mathrm{~mL} / \mathrm{min}, \lambda=254 \mathrm{nM}, \mathrm{t}_{\mathrm{R}}=21.3 \mathrm{~min}$, minor, $\mathrm{t}_{\mathrm{R}}=15.3$ 
min, major). Anal. Calcd for $\mathrm{C}_{19} \mathrm{H}_{20} \mathrm{O}_{3}: \mathrm{C}, 77.00 ; \mathrm{H}, 6.80$. Found: $\mathrm{C}, 76.89 ; \mathrm{H}, 6.78$. HRMS (EI) Calcd for $\mathrm{C}_{19} \mathrm{H}_{20} \mathrm{O}_{3}$ (M): 296.1407. Found: 296.1407.

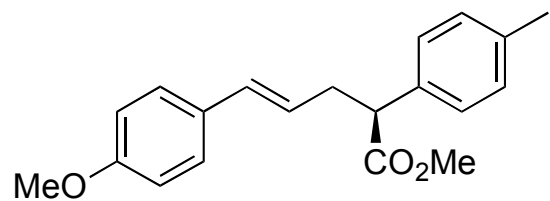

$5 d$

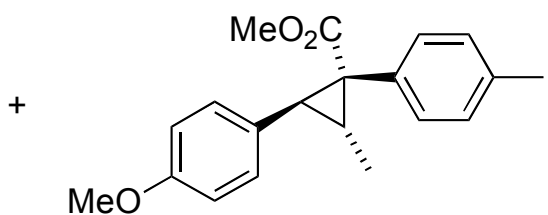

$6 d$

$(S, E)$-Methyl 5-(4-methoxyphenyl)-2-p-tolylpent-4-enoate (5d) and $(1 R, 2 S, 3 R)$-Methyl 2-(4-methoxyphenyl)-3-methyl-1-p-tolylcyclopropanecarboxylate (6d)

In a $25 \mathrm{~mL}$ round bottom flask trans-anethole $4(300 \mathrm{mg}, 2.00 \mathrm{mmol}, 1.00$ eq. $)$ and $\mathrm{Rh}_{2}(\mathrm{~S} \text {-DOSP })_{4}$ (38 mg, $0.02 \mathrm{mmol}, 0.01$ eq.) were dissolved in DMB (5 mL). Diazo 2d (768 mg, $4.00 \mathrm{mmol}, 2.00$ eq.) was stirred in distilled DMB (10 mL) under Ar for $5 \mathrm{~min}$ and then added via syringe pump for a $3 \mathrm{~h}$ period. The solution was then allowed to stir for an additional $2 \mathrm{~h}$. The solution was concentrated and crude ${ }^{1} \mathrm{H}$ NMR spectra indicated a product ratio of $(3: 1 ; \mathbf{5 d}: \mathbf{6 d})$. It was then purified by flash chromatography $\left(\mathrm{SiO}_{2}, 20 \% \mathrm{Et}_{2} \mathrm{O} /\right.$ pentane) to give $\mathbf{5 d}(354 \mathrm{mg}, 1.14 \mathrm{mmol}, 58 \%$ yield $)$ as a white solid and $\mathbf{6 d}$ (118 $\mathrm{mg}, 0.38 \mathrm{mmol}, 19 \%$ yield) as a white solid.

Compound 5d: White solid, mp $66{ }^{\circ} \mathrm{C} . \mathrm{R}_{\mathrm{f}}=0.30\left(20 \% \mathrm{Et}_{2} \mathrm{O} /\right.$ pentane $) .[\alpha]_{\mathrm{D}}{ }^{23}=-95.8(\mathrm{c}$ $\left.=1.0, \mathrm{CHCl}_{3}\right) .{ }^{1} \mathrm{H} \mathrm{NMR}\left(500 \mathrm{MHz}, \mathrm{CDCl}_{3}\right) \delta 7.20(\mathrm{~m}, 4 \mathrm{H}), 7.11(\mathrm{~d}, J=8 \mathrm{~Hz}, 2 \mathrm{H}), 6.78$ (d, $J=8 \mathrm{~Hz}, 2 \mathrm{H}), 6.35(\mathrm{~d}, J=16 \mathrm{~Hz}, 1 \mathrm{H}), 5.95(\mathrm{~m}, 1 \mathrm{H}), 3.74(\mathrm{~s}, 3 \mathrm{H}), 3.64(\mathrm{~m}, 4 \mathrm{H}), 2.92$ (m, 1H), $2.61(\mathrm{~m}, 1 \mathrm{H}) 2.30(\mathrm{~s}, 3 \mathrm{H}) .{ }^{13} \mathrm{C} \mathrm{NMR}\left(125 \mathrm{MHz}, \mathrm{CDCl}_{3}\right) \delta$ 173.9, 158.8, 136.9, 
135.6, 131.4, 130.1, 129.3, 127.7, 127.1, 124.7, 113.8, 55.1, 51.8, 51.4, 36.9, 20.9. IR (neat): $1734,1510,1248,1144 \mathrm{~cm}^{-1}$. HPLC analysis: 92\% ee $((R, R)$-Whelk, $1.0 i$-PrOH in hexane, $1.0 \mathrm{~mL} / \mathrm{min}, \lambda=254 \mathrm{nM}, \mathrm{t}_{\mathrm{R}}=26.1 \mathrm{~min}$, minor, $\mathrm{t}_{\mathrm{R}}=22.6 \mathrm{~min}$, major). Anal. Calcd for $\mathrm{C}_{20} \mathrm{H}_{20} \mathrm{O}_{3}$ : C, 77.39; H, 7.14. Found: C, 77.30; H, 7.14. HRMS (ESI) Calcd for $\left[\mathrm{C}_{20} \mathrm{H}_{22} \mathrm{O}_{3}\right]^{+} \mathrm{Na}\left(\mathrm{M}^{+} \mathrm{Na}\right)$ : 333.1461. Found: 333.1467.

Compound 6d: White solid, mp $61{ }^{\circ} \mathrm{C} . \quad \mathrm{R}_{\mathrm{f}}=0.36\left(20 \% \mathrm{Et}_{2} \mathrm{O} /\right.$ pentane). $[\alpha]_{\mathrm{D}}{ }^{23}=-94.4$ (c $\left.=1.0, \mathrm{CHCl}_{3}\right) .{ }^{1} \mathrm{H} \mathrm{NMR}\left(400 \mathrm{MHz}, \mathrm{CDCl}_{3}\right) 6.93(\mathrm{~d}, J=8 \mathrm{~Hz}, 2 \mathrm{H}), 6.87(\mathrm{~d}, J=8 \mathrm{~Hz}$, 2H), $6.66(\mathrm{~d}, J=8 \mathrm{~Hz}, 2 \mathrm{H}), 6.59(\mathrm{~d}, J=8 \mathrm{~Hz}, 2 \mathrm{H}), 3.69(\mathrm{~s}, 3 \mathrm{H}), 3.63(\mathrm{~s}, 3 \mathrm{H}), 2.99$ (d, $J=$ $8 \mathrm{~Hz}, 1 \mathrm{H}), 2.24(\mathrm{~s}, 3 \mathrm{H}), 2.13(\mathrm{~m}, 1 \mathrm{H}), 1.44(\mathrm{~d}, J=6 \mathrm{~Hz}, 3 \mathrm{H}) .{ }^{13} \mathrm{C}$ NMR $(75 \mathrm{MHz}$, $\left.\mathrm{CDCl}_{3}\right) \delta 172.6,157.9,136.3,133.4,131.1,129.2,128.9,128.6,113.1,55.1,55.0,42.6$, 37.2, 27.5, 21.1, 12.9. IR (neat): $1717,1516,1458,1252,1168 \mathrm{~cm}^{-1}$. HPLC analysis: $62 \%$ ee $\left((R, R)\right.$-Whelk, $0.5 i$-PrOH in hexane, $1.0 \mathrm{~mL} / \mathrm{min}, \lambda=254 \mathrm{nM}, \mathrm{t}_{\mathrm{R}}=34.0 \mathrm{~min}$, minor, $t_{R}=40.4$ min, major). Anal. Calcd for $\mathrm{C}_{20} \mathrm{H}_{20} \mathrm{O}_{3}: \mathrm{C}, 77.39 ; \mathrm{H}, 7.14$. Found: $\mathrm{C}$, 77.18; H, 7.08. HRMS (EI) Calcd for $\mathrm{C}_{20} \mathrm{H}_{22} \mathrm{O}_{3}$ (M): 310.1563 Found: 310.1569 .

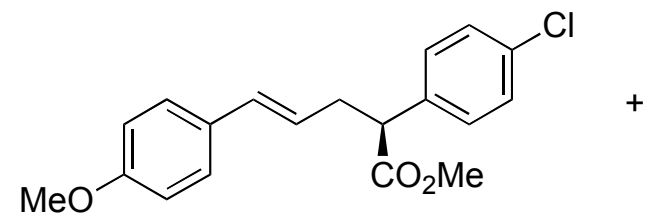

$5 e$

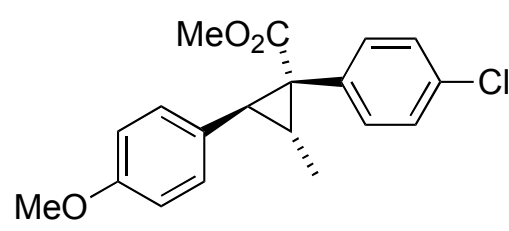

$6 e$

(S,E)-Methyl 2-(4-chlorophenyl)-5-(4-methoxyphenyl)pent-4-enoate (5e) and (1R,2S,3R)-Methyl 1-(4-chlorophenyl)-2-(4-methoxyphenyl)-3methylcyclopropanecarboxylate (6e): 
In a $25 \mathrm{~mL}$ round bottom flask trans-anethole $4(150 \mathrm{mg}, 1.00 \mathrm{mmol}, 1.00$ eq.) and $\mathrm{Rh}_{2}(S \text {-DOSP })_{4}$ (38 mg, $0.02 \mathrm{mmol}, 0.02$ eq.) were dissolved in DMB $(6 \mathrm{~mL})$. Diazo 2e (425 mg, 2.00 mmol, 2.00 eq.) was stirred in distilled $\mathrm{DMB}: \mathrm{CF}_{3} \mathrm{Ph}(5: 1 ; 12 \mathrm{~mL})$ under Ar for $5 \mathrm{~min}$ and then added via syringe pump for a $3 \mathrm{~h}$ period. The solution was then allowed to stir for an additional $2 \mathrm{~h}$, concentrated and the crude ${ }^{1} \mathrm{H}$ NMR spectrum indicated a product ratio of $(3: 1 ; 5 \mathbf{e}: \mathbf{6 e})$. The residue was purified by flash chromatography $\left(\mathrm{SiO}_{2}, 20 \% \mathrm{Et}_{2} \mathrm{O} /\right.$ pentane) to give $\mathbf{5 e}(153 \mathrm{mg}, 0.46 \mathrm{mmol}, 46 \%$ yield) as a white solid and $6 \mathrm{e}(60 \mathrm{mg}, 0.18 \mathrm{mmol}, 18 \%$ yield) as a white solid.

Compound 5e: White solid, mp $67{ }^{\circ} \mathrm{C} . \quad \mathrm{R}_{\mathrm{f}}=0.32\left(20 \% \mathrm{Et}_{2} \mathrm{O} /\right.$ pentane $) .[\alpha]_{\mathrm{D}}{ }^{23}=-71.4(\mathrm{c}$ $\left.=1.1, \mathrm{CHCl}_{3}\right) .{ }^{1} \mathrm{H}$ NMR $\left(500 \mathrm{MHz}, \mathrm{CDCl}_{3}\right) \delta 7.27(\mathrm{~m}, 6 \mathrm{H}), 6.81(\mathrm{~d}, J=8 \mathrm{~Hz}, 2 \mathrm{H}), 6.35$ (d, $J=16 \mathrm{~Hz}, 1 \mathrm{H}), 5.91(\mathrm{dt}, J=7 \mathrm{~Hz}, 16 \mathrm{~Hz}, 1 \mathrm{H}), 3.78(\mathrm{~s}, 3 \mathrm{H}), 3.65(\mathrm{~m}, 4 \mathrm{H}), 2.91(\mathrm{~m}$, 1H), $2.61(\mathrm{~m}, 1 \mathrm{H}) .{ }^{13} \mathrm{C} \mathrm{NMR}\left(125 \mathrm{MHz}, \mathrm{CDCl}_{3}\right) \delta 173.5,158.9,136.9,133.2,131.9$, $129.9,129.3,128.8,127.2,124.1,113.9,53.2,52.1,51.2,36.9$. IR (neat): 1737, 1604, 1518, 1438, 1252, 1167, 1092, 1034, 970, $826 \mathrm{~cm}^{-1}$. HPLC analysis: 92\% ee (OD-H, 0.5 $i$-PrOH in hexane, $1.0 \mathrm{~mL} / \mathrm{min}, \lambda=254 \mathrm{nM}, \mathrm{t}_{\mathrm{R}}=20.7 \mathrm{~min}$, minor, $\mathrm{t}_{\mathrm{R}}=23.1 \mathrm{~min}$, major). Anal. Calcd for $\mathrm{C}_{19} \mathrm{H}_{19} \mathrm{ClO}_{3}$ : C, 68.98; H, 5.79. Found: C, 69.09; H, 5.90. HRMS (EI) Calcd for $\mathrm{C}_{19} \mathrm{H}_{19} \mathrm{ClO}_{3}(\mathrm{M})$ : 330.1019 . Found: 330.1019 .

Compound 6e: White solid, mp $64{ }^{\circ} \mathrm{C} . \quad \mathrm{R}_{\mathrm{f}}=0.36\left(20 \% \mathrm{Et}_{2} \mathrm{O} /\right.$ pentane $) . \quad[\alpha]_{\mathrm{D}}^{23}=-28.8(\mathrm{c}$ $\left.=1.0, \mathrm{CHCl}_{3}\right) .{ }^{1} \mathrm{H} \mathrm{NMR}\left(400 \mathrm{MHz}, \mathrm{CDCl}_{3}\right) 7.09(\mathrm{~d}, J=8 \mathrm{~Hz}, 2 \mathrm{H}), 6.90(\mathrm{~d}, J=8 \mathrm{~Hz}$, 2H), $6.67(\mathrm{~d}, J=9 \mathrm{~Hz}, 2 \mathrm{H}), 6.61(\mathrm{~d}, J=9 \mathrm{~Hz}, 2 \mathrm{H}), 3.70(\mathrm{~s}, 3 \mathrm{H}), 3.63(\mathrm{~s}, 3 \mathrm{H}), 3.02(\mathrm{~d}, J$ $=8 \mathrm{~Hz}, 1 \mathrm{H}), 2.13(\mathrm{~m}, 1 \mathrm{H}), 1.45(\mathrm{~d}, J=6 \mathrm{~Hz}, 3 \mathrm{H}) .{ }^{13} \mathrm{C} \mathrm{NMR}\left(75 \mathrm{MHz}, \mathrm{CDCl}_{3}\right) \delta 171.9$, $158.1,135.2,132.7,128.9,128.6,128.0,113.3,55.1,52.3,42.2,37.4,27.5,12.7$, one 
carbon missing or overlapping. IR (neat): 1718, 1516, 1493, 1250, 1214, $1169 \mathrm{~cm}^{-1}$. HPLC analysis: $81 \%$ ee (OD-H, $0.5 i-\mathrm{PrOH}$ in hexane, $0.8 \mathrm{~mL} / \mathrm{min}, \lambda=254 \mathrm{nM}, \mathrm{t}_{\mathrm{R}}=$ 21.2 min, minor, $\mathrm{t}_{\mathrm{R}}=22.2$ min, major). Anal. Calcd for $\mathrm{C}_{19} \mathrm{H}_{19} \mathrm{ClO}_{3}: \mathrm{C}, 68.98 ; \mathrm{H}, 5.79$. Found: $\mathrm{C}, 68.79$; $\mathrm{H}, 5.69$. HRMS (EI) Calcd for $\mathrm{C}_{19} \mathrm{H}_{19} \mathrm{ClO}_{3}$ (M): 330.1017. Found: 330.1020.<smiles>COc1ccc(/C=C/C[C@H](C(C)=O)c2ccc(C(F)(F)F)cc2)cc1</smiles>

$5 f$

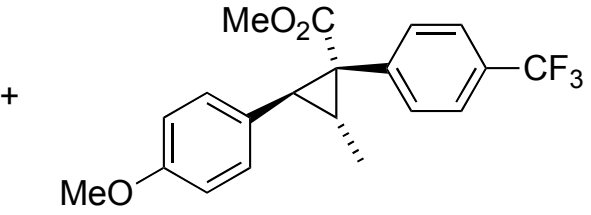

$6 f$

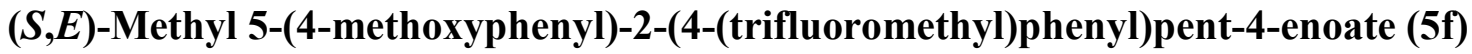
and (1R,2S,3R)-Methyl 2-(4-methoxyphenyl)-3-methyl-1-(4(trifluoromethyl)phenyl)cyclopropanecarboxylate (6f)

In a $25 \mathrm{~mL}$ round bottom flask trans-anethole $4(528 \mathrm{mg}, 3.50 \mathrm{mmol}, 1.00$ eq.) and $\mathrm{Rh}_{2}(S \text {-DOSP })_{4}(132 \mathrm{mg}, 0.07 \mathrm{mmol}, 0.02$ eq.) were dissolved in DMB (50 mL). Diazo 2f (1.73 g, $7.10 \mathrm{mmol}, 2.03$ eq.) was stirred in distilled $\mathrm{DMB}: \mathrm{CF}_{3} \mathrm{Ph}(5: 1 ; 20 \mathrm{~mL})$ under $\operatorname{Ar}$ for $5 \mathrm{~min}$. It was then added via syringe pump for a $3 \mathrm{~h}$ period. The solution was then allowed to stir for an additional $2 \mathrm{~h}$. The solution was concentrated and crude ${ }^{1} \mathrm{H}$ NMR spectra indicated a product ratio of $(2: 1 ; \mathbf{5 f}: \mathbf{6 f})$. It was then purified by flash chromatography $\left(\mathrm{SiO}_{2}, 20 \% \mathrm{Et}_{2} \mathrm{O} /\right.$ pentane) to give $\mathbf{5 f}$ (480 mg, $1.30 \mathrm{mmol}, 37 \%$ yield) as an oil and $\mathbf{6 f}$ (142 mg, $0.38 \mathrm{mmol}, 11 \%$ yield) as an oil. 
Compound 5f: Clear oil. $\mathrm{R}_{\mathrm{f}}=0.32\left(20 \% \mathrm{Et}_{2} \mathrm{O} /\right.$ pentane $) .[\alpha]_{\mathrm{D}}^{23}=-62.0\left(\mathrm{c}=1.0, \mathrm{CHCl}_{3}\right)$. ${ }^{1} \mathrm{H}$ NMR $\left(500 \mathrm{MHz}, \mathrm{CDCl}_{3}\right) \delta 7.58(\mathrm{~d}, J=8 \mathrm{~Hz}, 2 \mathrm{H}), 7.45(\mathrm{~d}, J=8 \mathrm{~Hz}, 2 \mathrm{H}), 7.22(\mathrm{~d}, J=$ $8 \mathrm{~Hz}, 2 \mathrm{H}), 6.81(\mathrm{~d}, J=8 \mathrm{~Hz}, 2 \mathrm{H}), 6.37(\mathrm{~d}, J=16 \mathrm{~Hz}, 1 \mathrm{H}), 5.91(\mathrm{~m}, 1 \mathrm{H}), 3.78(\mathrm{~m}, 4 \mathrm{H})$, $3.67(\mathrm{~s}, 3 \mathrm{H}), 2.96(\mathrm{~m}, 1 \mathrm{H}), 2.63(\mathrm{~m}, 1 \mathrm{H}) .{ }^{13} \mathrm{C} \mathrm{NMR}\left(75 \mathrm{MHz}, \mathrm{CDCl}_{3}\right)$ 173.2, 159.1, $142.5,132.2,129.9,129.7(\mathrm{q}, J=32 \mathrm{~Hz}), 128.4,127.3,125.6(\mathrm{q}, J=4 \mathrm{~Hz}), 124.1(\mathrm{q}, J=$ $270 \mathrm{~Hz}), 123.8,113.9,55.2,52.2,51.7,36.9$. IR (neat): 1736, 1608, 1511, 1437, 1324, 1247, 1161, 1122, 1068, 1035, 1019, 967, $838 \mathrm{~cm}^{-1}$. HPLC analysis: $80 \%$ ee (OD-H, 0.5 $i$-PrOH in hexane, $0.5 \mathrm{~mL} / \mathrm{min}, \lambda=254 \mathrm{nM}, \mathrm{t}_{\mathrm{R}}=43.1 \mathrm{~min}$, major, $\mathrm{t}_{\mathrm{R}}=52 \mathrm{~min}$, minor). Anal. Calcd for $\mathrm{C}_{20} \mathrm{H}_{19} \mathrm{~F}_{3} \mathrm{O}_{3}$ : C, 65.93; H, 5.26. Found: C, 65.78; H, 5.27. HRMS (ESI) Calcd for $\left[\mathrm{C}_{20} \mathrm{H}_{19} \mathrm{~F}_{3} \mathrm{O}_{3}\right]^{+} \mathrm{Na}\left(\mathrm{M}^{+} \mathrm{Na}\right): 387.1178$. Found: 387.1173 .

Compound 6f: Clear oil. $\mathrm{R}_{\mathrm{f}}=0.31\left(20 \% \mathrm{Et}_{2} \mathrm{O} /\right.$ pentane $) .[\alpha]_{\mathrm{D}}^{23}=-70.4\left(\mathrm{c}=1.0, \mathrm{CHCl}_{3}\right)$; ${ }^{1} \mathrm{H}$ NMR $\left(500 \mathrm{MHz}, \mathrm{CDCl}_{3}\right) 7.37$ (d, $\left.J=8 \mathrm{~Hz}, 2 \mathrm{H}\right), 7.09$ (d, $\left.J=8 \mathrm{~Hz}, 2 \mathrm{H}\right), 6.67$ (d, $J=9$ Hz, 2H), $6.62(\mathrm{~d}, J=9 \mathrm{~Hz}, 2 \mathrm{H}), 3.69$ (s, 3H), $3.64(\mathrm{~s}, 3 \mathrm{H}), 3.08$ (d, $J=8 \mathrm{~Hz}, 1 \mathrm{H}), 2.19$ $(\mathrm{m}, 1 \mathrm{H}), 1.48(\mathrm{~d}, J=7 \mathrm{~Hz}, 3 \mathrm{H}) .{ }^{13} \mathrm{C}$ NMR $\left(75 \mathrm{MHz}, \mathrm{CDCl}_{3}\right) \delta 171.6,158.2,140.8$, 131.7, 126.9, $128.2(\mathrm{q}, J=32 \mathrm{~Hz}), 124.8,124.6(\mathrm{q}, J=4 \mathrm{~Hz}), 124.1(\mathrm{q}, J=270 \mathrm{~Hz})$, 113.4, 55.1, 52.2, 42.5, 37.5, 27.4, 12.6. IR (neat): 1736, 1511, 1324, 1247, 1161, 1121, $1110,1068,1035,1019,838 \mathrm{~cm}^{-1}$. HPLC analysis: $72 \%$ ee (OD-H, $0.5 i$-PrOH in hexane, $0.5 \mathrm{~mL} / \mathrm{min}, \lambda=254 \mathrm{nM}, \mathrm{t}_{\mathrm{R}}=17.2 \mathrm{~min}$, major, $\mathrm{t}_{\mathrm{R}}=18.4 \mathrm{~min}$, minor). Anal. Calcd for $\mathrm{C}_{20} \mathrm{H}_{19} \mathrm{~F}_{3} \mathrm{O}_{3}$ : C, 65.93; H, 5.26. Found: C, 65.65; H, 5.26. HRMS (ESI) Calcd. for $\left[\mathrm{C}_{20} \mathrm{H}_{19} \mathrm{~F}_{3} \mathrm{O}_{3}\right]^{+} \mathrm{Na}\left(\mathrm{M}^{+} \mathrm{Na}\right)$ : 387.1167. Found: 387.1167 . 


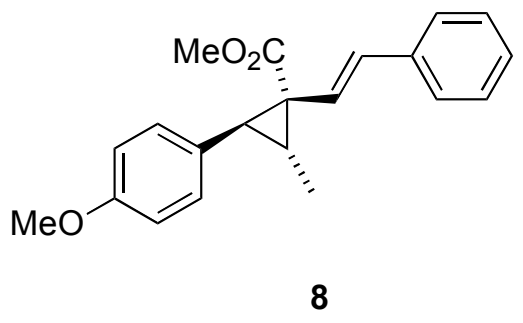

(1R, 2S, 3R, E)-Methyl 2-(4-methoxyphenyl)-3-methyl-1styrylcyclopropanecarboxylate (8)

In a dry $25 \mathrm{~mL}$ round bottom flask, trans-anethole $4(51 \mathrm{~mL}, 0.34 \mathrm{mmol}, 1.00$ eq.) and $\mathrm{Rh}_{2}(S \text {-DOSP })_{4}(13 \mathrm{mg}, 0.007 \mathrm{mmol}, 0.02$ eq. $)$ were dissolved in DMB (5 mL). Diazo 7 (136 mg, $0.68 \mathrm{mmol}, 2.00$ eq.) was stirred in distilled DMB (7 mL) under Ar for $5 \mathrm{~min}$. It was then added via syringe pump for a $3 \mathrm{~h}$ period. The solution was then allowed to stir for an additional $2 \mathrm{~h}$. The solution was concentrated and purified by flash chromatography $\left(\mathrm{SiO}_{2}, 20 \% \mathrm{Et}_{2} \mathrm{O} /\right.$ pentane $)$ to give $\mathbf{8}(67 \mathrm{mg}, 0.21 \mathrm{mmol}, 61 \%$ yield $)$ as a clear oil. $\quad \mathrm{R}_{\mathrm{f}}=0.43\left(20 \% \mathrm{Et}_{2} \mathrm{O} /\right.$ pentane $) . \quad[\alpha]_{\mathrm{D}}{ }^{23}=-123.0\left(\mathrm{c}=1.0, \mathrm{CHCl}_{3}\right) .{ }^{1} \mathrm{H} \mathrm{NMR}$ $\left(500 \mathrm{MHz}, \mathrm{CDCl}_{3}\right) \delta 7.22(\mathrm{t}, J=7 \mathrm{~Hz}, 2 \mathrm{H}), 7.15(\mathrm{~m}, 3 \mathrm{H}), 7.05(\mathrm{~d}, J=8 \mathrm{~Hz}, 2 \mathrm{H}), 6.76(\mathrm{~d}$, $J=8 \mathrm{~Hz}, 2 \mathrm{H}), 6.35(\mathrm{~d}, J=16 \mathrm{~Hz}, 1 \mathrm{H}), 5.96(\mathrm{~d}, J=16 \mathrm{~Hz}, 1 \mathrm{H}), 3.76(\mathrm{~m}, 6 \mathrm{H}), 2.96(\mathrm{~d}, J=$ $7 \mathrm{~Hz}, 1 \mathrm{H}), 2.08(\mathrm{~m}, 1 \mathrm{H}), 1.42(\mathrm{~d}, J=6 \mathrm{~Hz}, 3 \mathrm{H}) .{ }^{13} \mathrm{C} \mathrm{NMR}\left(75 \mathrm{MHz}, \mathrm{CDCl}_{3}\right) \delta 172.2$ $158.3,137.3,132.4,129.9,128.4,127.2,126.3,126.1,113.5,55.2,52.1,38.9,38.6,27.6$, 12.9, one carbon missing or overlapping. IR (neat): 1719, 1612, 1516, 1435, 1302, 1246, 1179, 1156, 1122, 1035, 963, 834, 744, $694 \mathrm{~cm}^{-1}$. HPLC analysis: 74\% ee (OD-H, 0.5\% $i-\mathrm{PrOH}$ in hexane, $1.0 \mathrm{~mL} / \mathrm{min}, \lambda=254 \mathrm{nM}, \mathrm{t}_{\mathrm{R}}=10.6 \mathrm{~min}$, minor, $\mathrm{t}_{\mathrm{R}}=12.3 \mathrm{~min}$, major). Anal. Calcd for $\mathrm{C}_{21} \mathrm{H}_{22} \mathrm{O}_{3}$ : C, 78.23; H, 6.88. Found: C, 77.93; H, 6.86. HRMS (ESI) Calcd for $\left[\mathrm{C}_{21} \mathrm{H}_{22} \mathrm{O}_{3}\right]^{+} \mathrm{Na}\left(\mathrm{M}^{+} \mathrm{Na}\right)$ : 345.1461 . Found: 345.1458 . 


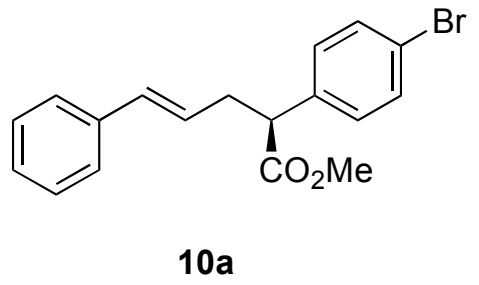

\section{(S,E)-Methyl 2-(4-bromophenyl)-5-phenylpent-4-enoate (10a)}

In a dry $25 \mathrm{~mL}$ round bottom flask trans- $\beta$-methyl styrene, 9a (435 mg, $3.60 \mathrm{mmol}, 1.00$ eq.) and $\mathrm{Rh}_{2}(S \text {-DOSP })_{4}(136 \mathrm{mg}, 0.072 \mathrm{mmol}, 0.02$ eq.) were dissolved in DMB (10 mL). Diazo 2a (1.87 g, $7.20 \mathrm{mmol}, 2.00$ eq.) was stirred in distilled DMB (20 mL) under Ar for $5 \mathrm{~min}$. It was then added via syringe pump for a $3 \mathrm{~h}$ period. The solution was then allowed to stir for an additional $2 \mathrm{~h}$. The solution was concentrated, dry-mounted on silica, and subjected to flash chromatography $\left(\mathrm{SiO}_{2}, 20 \% \mathrm{Et}_{2} \mathrm{O} /\right.$ pentane) to give 10a (634

$\mathrm{mg}, 1.84 \mathrm{mmol}, 51 \%$ yield $)$ as a clear oil. $\mathrm{R}_{\mathrm{f}}=0.45\left(20 \% \mathrm{Et}_{2} \mathrm{O} /\right.$ pentane $) .[\alpha]_{\mathrm{D}}{ }^{23}=-82.4$ $\left(\mathrm{c}=0.99, \mathrm{CHCl}_{3}\right) .{ }^{1} \mathrm{H}$ NMR $\left(500 \mathrm{MHz}, \mathrm{CDCl}_{3}\right) \delta 7.45(\mathrm{~m}, 2 \mathrm{H}), 7.23(\mathrm{~m}, 7 \mathrm{H}), 6.41(\mathrm{~d}, J$ $=16 \mathrm{~Hz}, 1 \mathrm{H}), 6.05(\mathrm{~m}, 1 \mathrm{H}), 3.66(\mathrm{~m}, 4 \mathrm{H}), 2.92(\mathrm{~m}, 1 \mathrm{H}), 2.63(\mathrm{~m}, 1 \mathrm{H}) .{ }^{13} \mathrm{C} \mathrm{NMR}(125$ $\left.\mathrm{MHz}, \mathrm{CDCl}_{3}\right) \delta 173.3,137.4,137.1,132.5,131.8,129.6,128.4,127.3,126.3,126.1$, 121.3, 52.1, 51.1, 36.8. IR (neat): 1735, 1488, 1160, 1011, 966, 745, $693 \mathrm{~cm}^{-1}$. HPLC analysis: $84 \%$ ee $\left((R, R)-\right.$ Whelk, $1 \% i-\mathrm{PrOH}$ in hexane, $1.0 \mathrm{~mL} / \mathrm{min}, \lambda=254 \mathrm{nM}, \mathrm{t}_{\mathrm{R}}=23.7$ min, major, $t_{R}=25.7$ min, minor). Anal. Calcd for $\mathrm{C}_{18} \mathrm{H}_{17} \mathrm{BrO}_{2}$ : C, 62.62; $\mathrm{H}, 4.96$. Found: C, 62.34; H, 5.07. HRMS (EI) Calcd for $\mathrm{C}_{18} \mathrm{H}_{17} \mathrm{BrO}_{2}$ (M): 344.0406. Found: 344.0411 . 


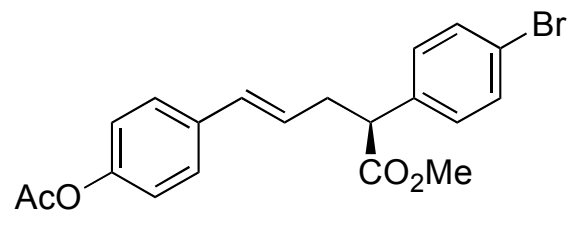

10b

\section{(S,E)-Methyl 2-(4-bromophenyl)-5-(4-(ethanoyloxy)phenyl)pent-4-enoate (10b)}

In a dry $25 \mathrm{~mL}$ round bottom flask (E)-4-(prop-1-enyl)phenyl ethanoate, $9 \mathbf{b}$ (435 mg, $3.60 \mathrm{mmol}, 1.00$ eq.) and $\mathrm{Rh}_{2}(S \text {-DOSP })_{4}(136 \mathrm{mg}, 0.072 \mathrm{mmol}, 0.02 \mathrm{eq}$.) were dissolved in DMB (10 mL). Diazo 2a (1.87 g, $7.20 \mathrm{mmol}, 2.00$ eq.) was stirred in distilled DMB $(20 \mathrm{~mL}$ ) under Ar for $5 \mathrm{~min}$. It was then added via syringe pump for a $3 \mathrm{~h}$ period. The solution was then allowed to stir for an additional $2 \mathrm{~h}$. The solution was concentrated, dry-mounted on silica, and subjected to flash chromatography $\left(\mathrm{SiO}_{2}, 20 \% \mathrm{Et}_{2} \mathrm{O} /\right.$ pentane $)$ to give $10 \mathrm{~b}(1.045 \mathrm{~g}, 2.59 \mathrm{mmol}, 72 \%$ yield $)$ as a clear oil. $\mathrm{R}_{\mathrm{f}}=0.45(20 \%$ $\mathrm{Et}_{2} \mathrm{O} /$ pentane $) .[\alpha]_{\mathrm{D}}^{23}=-12.2\left(\mathrm{c}=1.0, \mathrm{CHCl}_{3}\right) .{ }^{1} \mathrm{H}$ NMR $\left(500 \mathrm{MHz}, \mathrm{CDCl}_{3}\right) \delta 7.45(\mathrm{~d}, J$ $=8 \mathrm{~Hz}, 2 \mathrm{H}), 7.27(\mathrm{~d}, J=8 \mathrm{~Hz}, 2 \mathrm{H}), 7.20(\mathrm{~d}, J=8 \mathrm{~Hz}, 2 \mathrm{H}), 6.99(\mathrm{~d}, J=8 \mathrm{~Hz}, 2 \mathrm{H}), 6.38$ (d, $J=16 \mathrm{~Hz}, 1 \mathrm{H}), 6.08(\mathrm{~m}, 1 \mathrm{H}), 3.65(\mathrm{~m}, 4 \mathrm{H}), 2.92(\mathrm{~m}, 1 \mathrm{H}), 2.62(\mathrm{~m}, 1 \mathrm{H}), 2.28(\mathrm{~s}, 3 \mathrm{H})$. ${ }^{13} \mathrm{C}$ NMR $\left(75 \mathrm{MHz}, \mathrm{CDCl}_{3}\right) \delta 173.3,169.4,149.8,137.3,134.9,131.8,131.6,129.6$, $126.9,126.5,121.5,121.4,52.1,51.1,36.7,21.0$. IR (neat): 1756, 1734, 1506, 1211, 1193, 1164, $1011 \mathrm{~cm}^{-1}$. HPLC analysis: $86 \%$ ee $((S, S)$-Whelk, $1.0 \% i$-PrOH in hexane, $1.0 \mathrm{~mL} / \mathrm{min}, \lambda=254 \mathrm{nM}, \mathrm{t}_{\mathrm{R}}=38.2 \mathrm{~min}$, minor, $\mathrm{t}_{\mathrm{R}}=44.8 \mathrm{~min}$, major). HRMS (EI) Calcd for $\left[\mathrm{C}_{20} \mathrm{H}_{19} \mathrm{BrO}_{4}\right]^{+}\left(\mathrm{M}^{+}\right)$: 402.0461. Found 402.0460. 
<smiles>COc1cc(/C=C/C[C@H](C(C)=O)c2ccc(Br)cc2)cc(OC)c1OC</smiles>

$10 c$<smiles>COc1cc([C@@H]2[C@@H](C)[C@]2(C(C)=O)c2ccc(Br)cc2)cc(OC)c1OC</smiles>

11c

(S,E)-Methyl 2-(4-bromophenyl)-5-(3,4,5-trimethoxyphenyl)pent-4-enoate (10c) and $(1 R, 2 R, 3 S)$-Methyl 1-(4-bromophenyl)-2-methyl-3-(3,4,5,-trimethoxyphenyl) cyclopropanecarboxylate (11c)

In a $25 \mathrm{~mL}$ round bottom flask the $(E)$-1,2,3-trimethoxy-5-(prop-1-enyl)benzene, 9c (150 mg, $1.00 \mathrm{mmol}, 1.00$ eq. $)$ and $\mathrm{Rh}_{2}(S \text {-DOSP })_{4}(38 \mathrm{mg}, 0.02 \mathrm{mmol}, 0.02$ eq. $)$ were dissolved in DMB (6 mL). Diazo 2a (508 mg, $2.00 \mathrm{mmol}, 2.00$ eq.) was stirred in distilled DMB (12 mL) under Ar for $5 \mathrm{~min}$. It was then added via syringe pump for a $3 \mathrm{~h}$ period. The solution was then allowed to stir for an additional $2 \mathrm{~h}$. The solution was concentrated and the crude reaction mixture indicated $(3: 1 ; \mathbf{1 0 c}: \mathbf{1 1 c})$. The residue was purified by flash chromatography $\left(\mathrm{SiO}_{2}, 20 \% \mathrm{Et}_{2} \mathrm{O} /\right.$ pentane) to give a clear oil 10c $(278$ mg, $0.64 \mathrm{mmol}, 64 \%$ yield) and clear oil 11c (87 mg, $0.2 \mathrm{mmol}, 20 \%$ yield).

Compound 10c: Clear oil. $\mathrm{R}_{\mathrm{f}}=0.17\left(60 \% \mathrm{Et}_{2} \mathrm{O} /\right.$ pentane $) . \quad[\alpha]_{\mathrm{D}}^{23}=-171.2(\mathrm{c}=1.0$, $\left.\mathrm{CHCl}_{3}\right) .{ }^{1} \mathrm{H} \mathrm{NMR}\left(500 \mathrm{MHz}, \mathrm{CDCl}_{3}\right) \delta 7.45(\mathrm{~d}, J=8 \mathrm{~Hz}, 2 \mathrm{H}), 7.21(\mathrm{~d}, J=8 \mathrm{~Hz}, 2 \mathrm{H})$, $6.51(\mathrm{~s}, 2 \mathrm{H}), 6.34(\mathrm{~d}, J=16 \mathrm{~Hz}, 1 \mathrm{H}), 5.99(\mathrm{~m}, 1 \mathrm{H}), 3.84(\mathrm{~m}, 9 \mathrm{H}), 3.67(\mathrm{~m}, 4 \mathrm{H}), 2.95(\mathrm{~m}$, 1H), $2.62(\mathrm{~m}, 1 \mathrm{H}) .{ }^{13} \mathrm{C} \mathrm{NMR}\left(75 \mathrm{MHz}, \mathrm{CDCl}_{3}\right) \delta 173.3,153.2,137.4,132.8,132.5$, $131.7,129.6,125.7,121.3,109.3,60.8,56.0,52.1,51.1,36.7$, one carbon missing or overlapping. IR (neat): $1733,1581,1237,1156,1125,1010 \mathrm{~cm}^{-1}$. HPLC analysis: $88 \%$ ee $\left((R, R)\right.$-Whelk, $10 \% i$-PrOH in hexane, $0.5 \mathrm{~mL} / \mathrm{min}, \lambda=254 \mathrm{nM}, \mathrm{t}_{\mathrm{R}}=25.0 \mathrm{~min}$, minor, 
$t_{R}=27.6$ min, major). Anal. Calcd for $\mathrm{C}_{21} \mathrm{H}_{23} \mathrm{BrO}_{5}: \mathrm{C}, 57.94 ; \mathrm{H}$, 5.33. Found: $\mathrm{C}, 57.89$; $\mathrm{H}$, 5.25. HRMS (EI) Calcd for $\mathrm{C}_{21} \mathrm{H}_{23} \mathrm{BrO}_{5}(\mathrm{M}): 434.0723$ Found: 434.0724 .

Compound 11c: Clear oil. $\mathrm{R}_{\mathrm{f}}=0.20\left(60 \% \mathrm{Et}_{2} \mathrm{O} /\right.$ pentane $) . \quad[\alpha]_{\mathrm{D}}{ }^{23}=-32.0(\mathrm{c}=0.5$, $\left.\mathrm{CHCl}_{3}\right) .{ }^{1} \mathrm{H} \mathrm{NMR}\left(500 \mathrm{MHz}, \mathrm{CDCl}_{3}\right) \delta 7.32(\mathrm{~d}, J=8 \mathrm{~Hz}, 2 \mathrm{H}), 6.92(\mathrm{~d}, J=8 \mathrm{~Hz}, 2 \mathrm{H})$, $5.90(\mathrm{~s}, 2 \mathrm{H}), 3.77(\mathrm{~s}, 3 \mathrm{H}), 3.67(\mathrm{~s}, 3 \mathrm{H}), 3.61(\mathrm{~s}, 6 \mathrm{H}), 3.00(\mathrm{~d}, J=7 \mathrm{~Hz}, 1 \mathrm{H}), 2.12(\mathrm{~m}, 1 \mathrm{H})$, $1.48(\mathrm{~d}, J=7 \mathrm{~Hz}, 3 \mathrm{H}) .{ }^{13} \mathrm{C} \mathrm{NMR}\left(75 \mathrm{MHz}, \mathrm{CDCl}_{3}\right) \delta 171.7,152.6,135.8,133.1,132.2$, $131.1,121.1,105.1,60.8,55.9,52.4,42.7,38.1,28.5,12.7$, one carbon missing or overlapping. IR (neat): 1718, 1588, 1245, 1199, 1126, 1010, $722 \mathrm{~cm}^{-1}$. HPLC analysis: $87 \%$ ee $\left((R, R)\right.$-Whelk, $10 \% i-\mathrm{PrOH}$ in hexane, $0.5 \mathrm{~mL} / \mathrm{min}, \lambda=254 \mathrm{nM}, \mathrm{t}_{\mathrm{R}}=13.79 \mathrm{~min}$, minor, $t_{R}=19.97$ min, major). Anal. Calcd for $\mathrm{C}_{21} \mathrm{H}_{23} \mathrm{BrO}_{5}$ : C, 57.94; H, 5.33. Found: $\mathrm{C}$, 57.91; $\mathrm{H}, 5.32$. HRMS (EI) Calcd for $\mathrm{C}_{21} \mathrm{H}_{23} \mathrm{BrO}_{5}$ (M): 434.0723. Found: 434.0724.

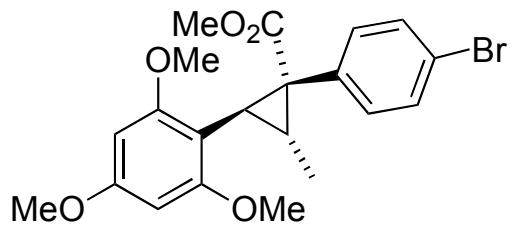

$11 d$

(1R, 2R, 3S)-Methyl 1-(4-bromophenyl)-2-methyl-3-(2,4,6-trimethoxyphenyl) cyclopropanecarboxylate (11d)

In a $25 \mathrm{~mL}$ round bottom flask (E)-1,3,5-trimethoxy-2-(prop-1-enyl)benzene, 9d (208 $\mathrm{mg}, 1.00 \mathrm{mmol}, 1.00$ eq. $)$ and $\mathrm{Rh}_{2}(S \text {-DOSP })_{4}(38 \mathrm{mg}, 0.02 \mathrm{mmol}, 0.02$ eq. $)$ were dissolved in DMB (6 mL). Diazo 2a (508 mg, $2.00 \mathrm{mmol}, 2.00$ eq.) was stirred in distilled DMB (12 mL) under Ar for $5 \mathrm{~min}$. It was then added via syringe pump for a $3 \mathrm{~h}$ 
period. The solution was then allowed to stir for an additional $2 \mathrm{~h}$. The solution was concentrated and purified by flash chromatography $\left(\mathrm{SiO}_{2}, 20 \% \mathrm{Et}_{2} \mathrm{O} /\right.$ pentane) to give 11d (325mg, $0.74 \mathrm{mmol}, 74 \%$ yield) as a clear oil. $\mathrm{R}_{\mathrm{f}}=0.17\left(20 \% \mathrm{Et}_{2} \mathrm{O} /\right.$ pentane $) .[\alpha]_{\mathrm{D}}{ }^{23}$ $=-71.6\left(\mathrm{c}=1.0, \mathrm{CHCl}_{3}\right) .{ }^{1} \mathrm{H}$ NMR $\left(400 \mathrm{MHz}, \mathrm{CDCl}_{3}\right) \delta 7.19(\mathrm{~d}, J=11 \mathrm{~Hz}, 2 \mathrm{H}), 6.90(\mathrm{~d}$, $J=11 \mathrm{~Hz}, 2 \mathrm{H}), 5.91(\mathrm{~s}, 2 \mathrm{H}), 3.71(\mathrm{~s}, 3 \mathrm{H}), 3.67(\mathrm{~s}, 3 \mathrm{H}), 3.62(\mathrm{~s}, 6 \mathrm{H}), 2.84(\mathrm{~m}, 2 \mathrm{H}), 1.42$ $(\mathrm{d}, J=7 \mathrm{~Hz}, 3 \mathrm{H}) .{ }^{13} \mathrm{C} \mathrm{NMR}\left(75 \mathrm{MHz}, \mathrm{CDCl}_{3}\right) \delta 172.9,160.1,159.7,137.4,131.7,130.3$, 120.1, 105.4, 90.7, 55.5, 55.1, 52.0, 38.9, 31.5, 27.7, 13.4. IR (neat): 1716, 1608, 1588, $1455,1434,1413,1331,1256,1226,1204,1152,1135,1120,1063,1039,1010,811,779$ $\mathrm{cm}^{-1}$. HPLC analysis: $87 \%$ ee $((R, R)-\mathrm{Whelk}, 2 \% i-\mathrm{PrOH}$ in hexane, $1.0 \mathrm{~mL} / \mathrm{min}, \lambda=254$ $\mathrm{nM}, \mathrm{t}_{\mathrm{R}}=35.6$ min, minor, $\mathrm{t}_{\mathrm{R}}=40.3$ min, major). Anal. Calcd for $\mathrm{C}_{21} \mathrm{H}_{23} \mathrm{BrO}_{5}: \mathrm{C}, 57.94$; H, 5.33. Found: C, 58.20; H, 5.45. HRMS (EI) Calcd for $\mathrm{C}_{21} \mathrm{H}_{23} \mathrm{BrO}_{5}$ (M): 434.0726. Found: 434.0726.

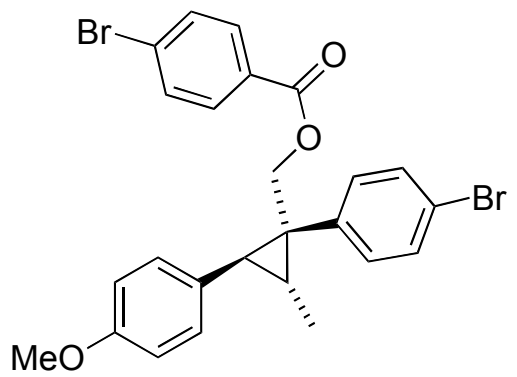

12

((1R,2S,3R)-1-(4-Bromophenyl)-2-(4-methoxyphenyl)-3-methylcyclopropyl)methyl 4-bromobenzoate (12)

Recrystalization of cyclopropane $\mathbf{6 a}$ by dissolving in diethyl ether, and then placed in a larger sealed vessel containing pentane, which diffused into solution to give colorless 
prisms. The solid was racemic, while the solution contained enantiomerically pure cyclopropane determined by HPLC analysis: $>99 \%$ ee $((R, R)$-Whelk, $0.5 i$-PrOH in hexane, $1.0 \mathrm{~mL} / \mathrm{min}, \lambda=254 \mathrm{nM}, \mathrm{t}_{\mathrm{R}}=6.6 \mathrm{~min}$, minor, $\mathrm{t}_{\mathrm{R}}=8.8 \mathrm{~min}$, major). $\mathrm{LiAlH}_{4}$ $(0.0186 \mathrm{~g}, 0.48 \mathrm{mmol}, 1.5$ eq. $)$ was added to a solution of enantiomerically pure cyclopropane $6 \mathrm{a}(0.1224 \mathrm{~g}, 0.32 \mathrm{mmol}, 1.0 \mathrm{eq}$.$) in \mathrm{THF}(20 \mathrm{~mL})$ at $0{ }^{\circ} \mathrm{C}$. The reaction mixture was stirred for 30 minutes. Diethyl ether $(25 \mathrm{~mL})$ and water $(50 \mathrm{~mL})$ were added, and the mixture was shaken and separated. The aqueous layer was further extracted with an additional portion of diethyl ether. The combined organic fractions were dried with $\mathrm{MgSO}_{4}$ and concentrated in vacuo. This residue was dissolved in $\mathrm{CH}_{2} \mathrm{Cl}_{2}(25 \mathrm{~mL})$ along with 4-bromobenzoic acid $(0.128 \mathrm{~g}, 0.64 \mathrm{mmol}, 2.0$ eq.) and DMAP (3 crystals, catalytic). The solution was cooled to $0{ }^{\circ} \mathrm{C}$ in an ice/water bath. Once cooled to $0{ }^{\circ} \mathrm{C}$, the dicyclohexylcarbodiimide $\left(1.0 \mathrm{M}^{\text {in }} \mathrm{CH}_{2} \mathrm{Cl}_{2} 0.64 \mathrm{~mL}, 0.64\right.$ mmol, 2.0 eq.) was added and stirred for $16 \mathrm{~h}$, while warming up to room temperature overnight. The mixture was filtered through a plug of cotton and the filtrate was washed with water. The organic fraction was dried with $\mathrm{MgSO}_{4}$ and concentrated in vacuo. The residue was purified by flash chromatography on silica gel using $5 \% \mathrm{Et}_{2} \mathrm{O} /$ pentane as the eluent to give $12(0.0899 \mathrm{~g}, 0.169 \mathrm{mmol}, 52 \%$ yield $)$ as a white solid, $\mathrm{mp} 127-129^{\circ} \mathrm{C} . \mathrm{R}_{\mathrm{f}}$ $=0.42\left(20 \% \mathrm{Et}_{2} \mathrm{O} /\right.$ pentane $) . \quad[\alpha]_{\mathrm{D}}{ }^{23}=+117.6\left(\mathrm{c}=1.0, \mathrm{CHCl}_{3}\right) . \quad{ }^{1} \mathrm{H}$ NMR $(500 \mathrm{MHz}$, $\left.\mathrm{CDCl}_{3}\right) \delta 7.80(\mathrm{~d}, J=9 \mathrm{~Hz}, 2 \mathrm{H}), 7.56(\mathrm{~d}, J=9 \mathrm{~Hz}, 2 \mathrm{H}), 7.27(\mathrm{~d}, J=9 \mathrm{~Hz}, 2 \mathrm{H}), 6.96(\mathrm{~d}, J$ $=9 \mathrm{~Hz}, 2 \mathrm{H}), 6.64(\mathrm{~s}, 4 \mathrm{H}), 4.73(\mathrm{~d}, J=12 \mathrm{~Hz}, 1 \mathrm{H}), 4.41(\mathrm{~d}, J=12 \mathrm{~Hz}, 1 \mathrm{H}), 3.72(\mathrm{~s}, 3 \mathrm{H})$, $2.14(\mathrm{~d}, J=6 \mathrm{~Hz}, 1 \mathrm{H}), 1.87$ (quint, $J=6 \mathrm{~Hz}, 1 \mathrm{H}), 1.47(\mathrm{~d}, J=6 \mathrm{~Hz}, 3 \mathrm{H}) .{ }^{13} \mathrm{C}$ NMR $(75$ $\left.\mathrm{MHz}, \mathrm{CDCl}_{3}\right) \delta 165.6,158.0,138.9,132.8,131.8,131.1,131.0,129.6,129.1,128.5$, 128.1, 120.7, 113.3, 70.2, 55.1, 37.2, 35.3, 28.9, 14.0. IR (neat): 1717, 1612, 1589, 1515, 
$1490,1466,1397,1267,1248,1173,1115,1100,1069,1037,1011,847,829,756,739$ $\mathrm{cm}^{-1}$. Anal. Calcd for $\mathrm{C}_{25} \mathrm{H}_{22} \mathrm{Br}_{2} \mathrm{O}_{3}: \mathrm{C}, 56.63 ; \mathrm{H}, 4.18$. Found: $\mathrm{C}, 56.73 ; \mathrm{H}, 4.21$. HRMS (EI) Calcd for $\mathrm{C}_{25} \mathrm{H}_{22} \mathrm{Br}_{2} \mathrm{O}_{3}$ (M): 527.9930. Found 527.9929. 


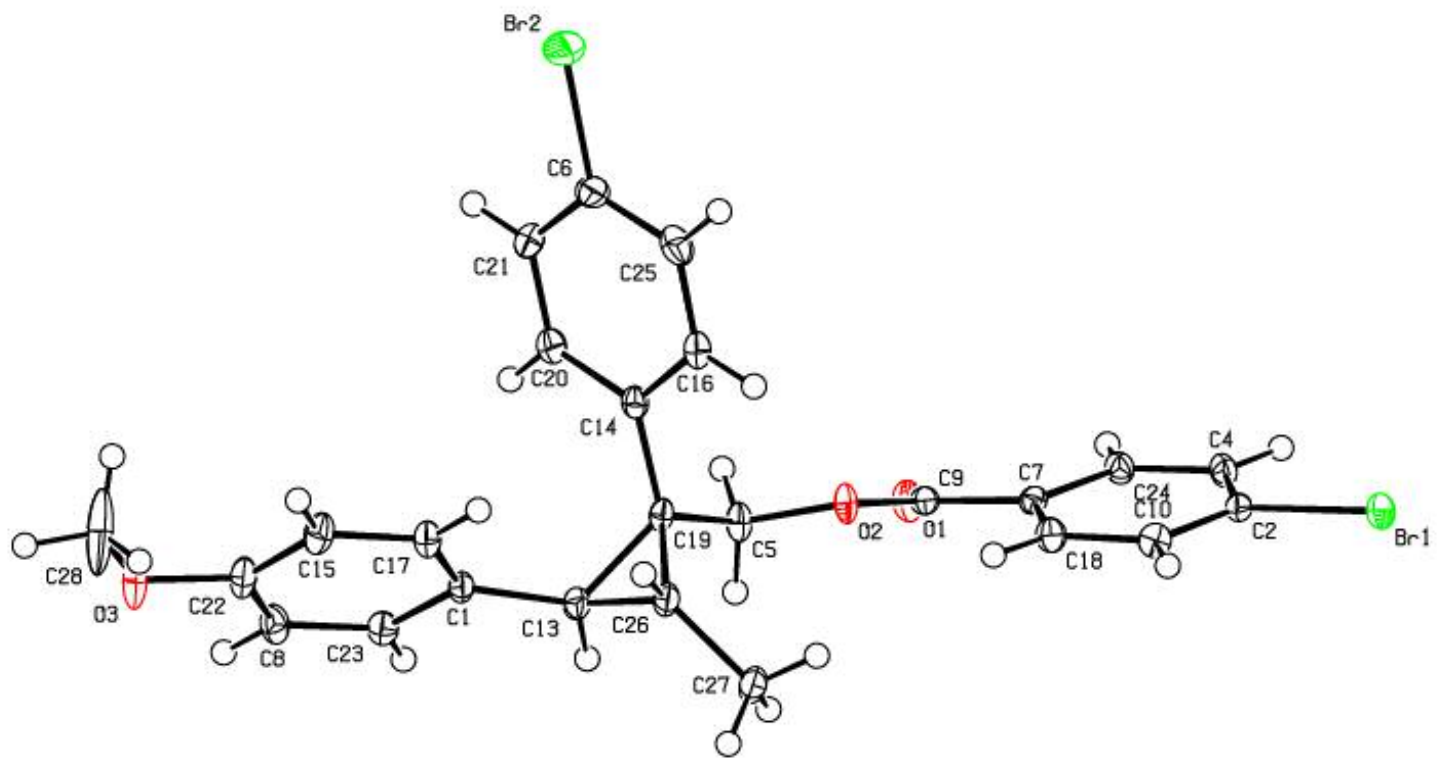

Fig.1. ORTEP plot and label scheme for 12. Displacement ellipsoids are drawn at the $50 \%$ probability level.

This compound was recrystallized by dissolving in diethyl ether, and then placed in a larger sealed vessel containing pentane, which diffused into solution to give colorless prisms.

References: 
1 Davies, H. M. L.; Bruzinski, P. R.; Lake, D. H.; Kong, N.; Fall, M. J. J. Am. Chem. Soc. 1996, 118, 6897.

2 Liu, J.-T.; Jang, Y.-J.; Shih, Y.-K.; Hu, S.-R.; Chu, C.-M.; Yao, C.-F. J. Org. Chem. 2001, 66, 6021.

3 Davies, H. M. L.; Hansen, T.; Churchill, M. R. J. Am. Chem. Soc. 2000, 122, 3063.

4 Baum, J. S.; Shook, D. A.; Davies, H. M. L.; Smith, H. D. Synth. Commun. 1987, 17, 1709-16.

5 Davies, H. M. L.; Venkataramani, C. Org. Lett. 2003, 5, 1403

6 Ni, A.; France, J. E.; Davies, H. M. L. J. Org. Chem. 2006, 71, 5594-5598.

7 Davies, H. M. L.; Clark, T. J.; Smith, H. D. J. Org. Chem. 1991, 56, 3817.

8 Engler, T. A.; Chai, W.; LaTessa, K. O. J. Org. Chem. 1996, 61, 9297.

9 Sharma, A.; Joshi, B. P.; Sinha, A. K. Bull. Chem. Soc. Jpn. 2004, 77, 2231. 


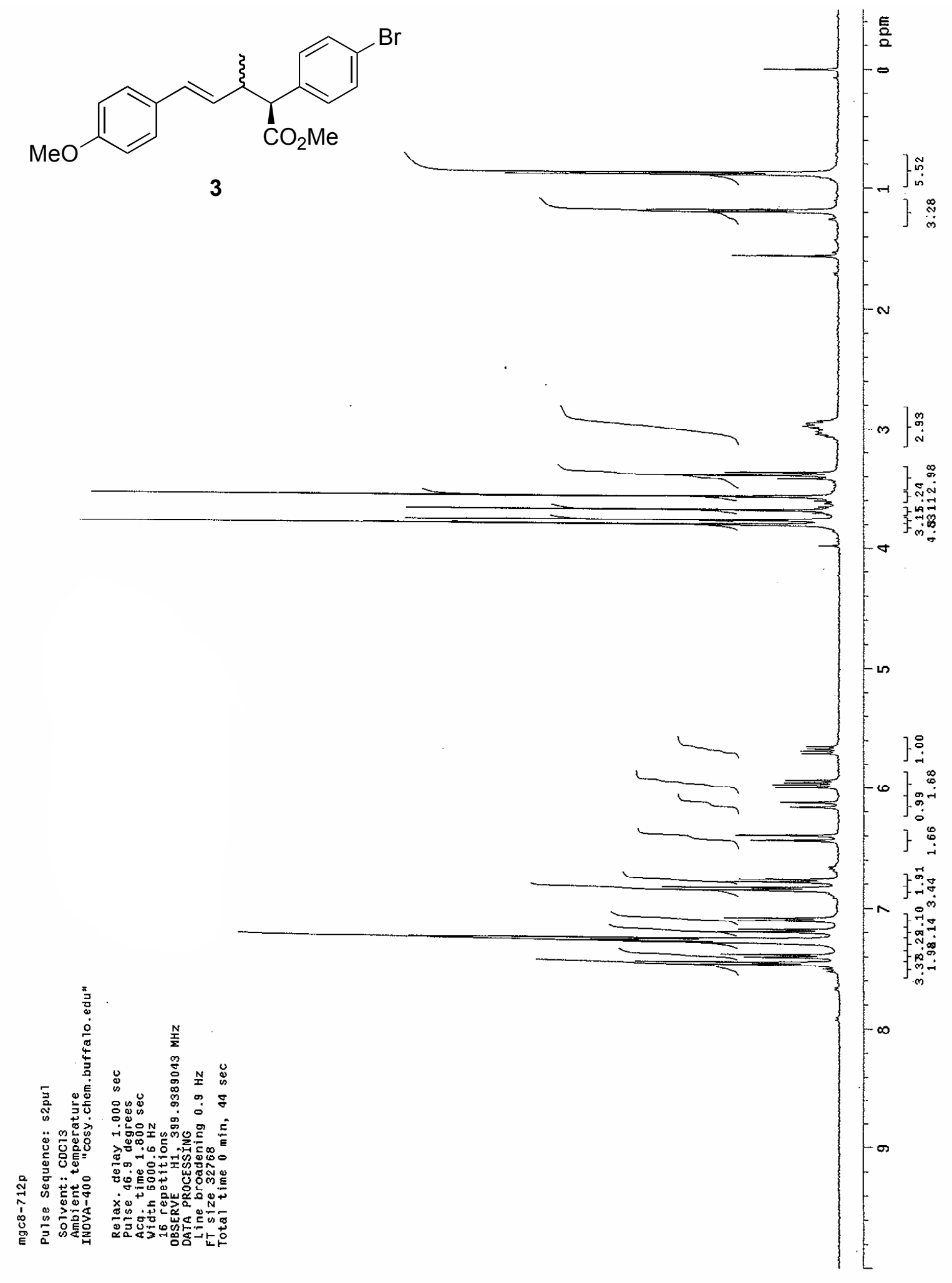




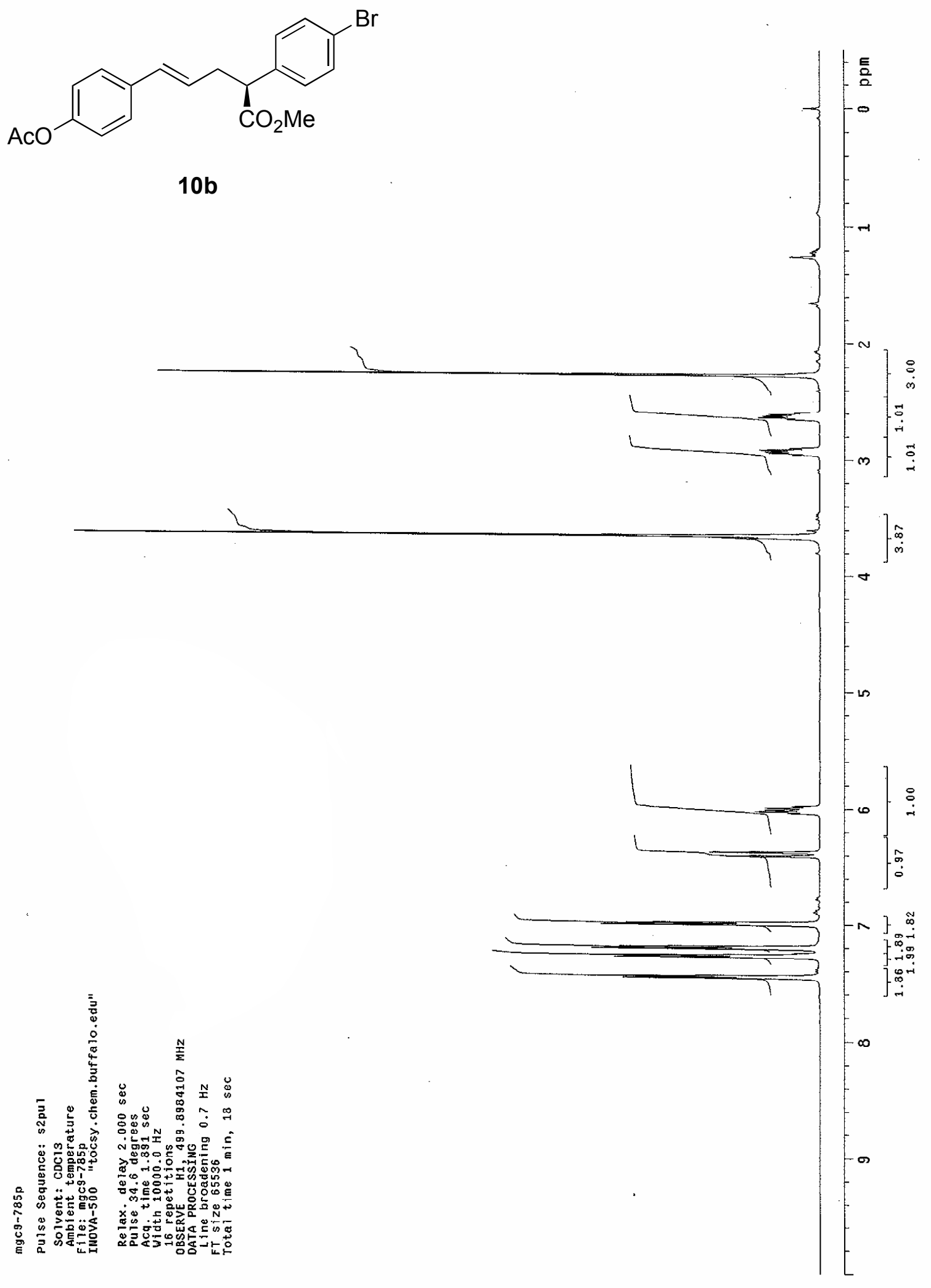

